- 'Anxiety and depression in adolescents and young adults"3 - Of particular concern is the alarming apparent rise of these problems in girls and young women. A 2017 UK practice-based study of self-harm showed a $68 \%$ rise in incidence in girls aged 13-16 years between 2011-2014.

- 'Patients' descriptions of the relation between physical symptoms and negative emotions'4 - 'Primary care guidelines for the management of persistent, often "medically unexplained", physical symptoms encourage GPs to discuss with patients how these symptoms relate to negative emotions.

- 'Medically unexplained symptoms ${ }^{5}$ - Most GPs labelled the presented symptoms as medically unexplained soon after the start of the consultation.

Taking into account the recent report on the 2018/2019 Public Health England review of prescribed medicines associated with dependence and withdrawal, ${ }^{6}$ the links become clear. Many patients who may suffer 'common mental health disorders', and those who suffer economic hardship and commonplace adversities, are prescribed drugs for 'depression', 'anxiety', 'trauma', etc. - which carry underplayed risks of dependence and withdrawal. Many such patients go on to experience all manner of distressing 'unexplained' physical and psychological symptoms. Some of these 'unexplained' symptoms can be very severe indeed, including drug-induced akathisia, which can lead to self-harm and suicide. Antidepressants land other prescribed medicines) can cause akathisia - especially at certain times of dose change or even after withdrawal - and this serious adverse drug reaction is frequently misdiagnosed as 'anxiety' and 'restlessness', with sometimes tragic consequences.?

Language matters very greatly. topics that are more closel interrelated than first appears, and the relationship is crucial for GPs to recognise.

As you say, language matters and 'The chances of a successful outcome are less good when ... the consultation includes topics such as common mental health disorders and the highly contested territories of persistent but "medically" unexplained symptoms and maladaptive responses to commonplace adversities:"

- 'Predicting and preventing relapse of depression in primary care ${ }^{2}$ - 'There has been a shift in the understanding of depression as a discrete or episodic illness to being considered a long-term relapsing-remitting condition with possibly incomplete recovery between episodes... descriptions of the relation between physical symptoms and negative emotions: a qualitative analysis of primary care consultations. Br J Gen Pract 2020; DOI: https://doi.org/10.3399/bjgp19X707369

5. Houwen J, Lucassen PLBJ, Dongelmans S, et al. Medically unexplained symptoms: time to and triggers for diagnosis in primary care consultations. Br J Gen Pract 2020; DOI: https:// doi.org/10.3399/bjgp20X707825.

6. Public Health England. Prescribed medicines review what this report means for patients. 2019. https:// unw.gov.uk/government/publications/prescribedmedicines-review-report laccessed 11 Mar 2020).

7. Gehrki KK. Rapid Response: Re: The gaps in our knowledge. BMJ 2020; 368: m161. www.bmj.com/ content/368/bmj.m161/rr-3 (accessed 11 Mar 2020).

DOI: https://doi.org/10.3399/bjgp20X709013

\section{Urgent referral of SSNHL to ENT}

The paper from ENT in Gloucester makes slightly depressing reading by advising that GPs should ignore the NICE recommendation to refer sudden hearing loss urgently to ENT because ENT departments can't offer this service.'

I would strongly recommend reading the full NICE Guideline ${ }^{2}$ section 11, pages 137-174, in particular sections 11.2 and 11.3, which show the extensive evidence surveyed and debated by the committee. Evidence was scarce, especially for oral steroids, which made it difficult to draft a recommendation for routes of administration.

A key issue is that five studies showed clinical benefit from intratympanic (IT) steroids for patients refractory to oral or intravenous steroids.

When administration of both oral and IT steroids was compared with either route alone, the committee commented on the clinical benefit of dual administration for recovery, PTA scores, and speech discrimination scores. There was uncertainty about the optimal route and timing (first or second line) owing to the limited number and quality of the studies.

It noted that practice varies considerably between centres and expressed concern about any delay in offering treatment.

Oral steroids are certainly the current favoured first-line treatment, and GPs should not delay starting them. I believe it is advisable to then contact their preferred ENT department as soon as practicable to agree a plan of action for follow-up and possible IT therapy in the event of failure of oral steroids. 
A 'non-urgent' referral that might cause several weeks' delay is not appropriate.

Ted Leverton,

Retired GP, RCGP Clinical Advisor.

Email: ted.levertondoutlook.com

\section{Competing interests}

Ted Leverton was a member of the NICE Guideline Committee on Adult Hearing Loss mentioned above.

\section{REFERENCES}

1. Ojha S, Henderson A, Bennett W. Clark M. Sudden sensorineural hearing loss and bedside phone testing: a guide for primary care. Br J Gen Pract 2020; DOI: https://doi.org/10.3399/bjgp20X708761.

2. National Institute for Health and Care Excellence. Hearing loss in adults: assessment and management. NG98. 2018. www.nice.org. uk/guidance/ng98/evidence/full-guidelinepdf-4852693117 (accessed 11 Mar 2020).

\section{Our prescription for climate change: reduce and recycle inhalers!}

I applaud the authors for their timely insights into the environmental impact of the myriad of inhalers we prescribe. ${ }^{1}$ I am sure if we are honest each of us has a handful of patients using more than 42 salbutamol metered dose inhalers (MDIs) per year - a figure that equates to the greenhouse gas from one car per year. ${ }^{1}$ Perhaps we should cycle to work until we have completed a salbutamol overuse audit?

Joking apart, it does seem that reducing and recycling MDls ought to be a priority given our current climate emergency. So on taking the authors' advice I was dismayed to discover that the Complete the Cycle recycling scheme was no longer accepting new referrals due to lack of funding. GSK, who run the scheme, confirmed this, but today I met with their Government Affairs Director and made the following suggestions:

- Reduce MDls - include a leaflet in all MDI packaging with a QR code linking to a YouTube video on how to use the MDI, thus improving technique and reducing waste. Facilitate the switch to DPIs by funding clinician time for the necessary reviews.

- Recycle MDIs - re-open the Complete the Cycle scheme with government backing.
The flip side of the above leaflet in MDI packaging should have instructions on how to recycle the MDI at your local surgery or pharmacy via the Complete the Cycle scheme.

And for us clinicians? Perhaps prescribing targets should direct us to DPIs, but best of all - raise awareness with MDI users.

Gordon Macdonald,

GP, Regent Gardens Medical Practice.

Email: gordon.macdonaldlanhs.net

\section{REFERENCE}

1. Mikolasch TA, Stadler $\mathrm{Cl}$. Our prescription for climate change: reduce and recycle inhalers! $\mathrm{Br}$ J Gen Pract 2020; DOI: https://doi.org/10.3399/ bjgp20X707717.

DOI: https://doi.org/10.3399/bjgp20X709037

\section{Building research capacity in primary care}

A recent $B J G P$ editorial ${ }^{1}$ highlights the importance of primary care research and argues that health systems should invest in the academics and research practice networks that provide the evidence." However, numerous barriers to participation in clinical research exist, particularly in the UK primary care setting where GPs are under immense pressure due to increased workloads and a dwindling workforce. ${ }^{2}$ Consequently, GPs may experience difficulty conducting research alongside clinical duties due to time constraints, competing interests, and a need to carefully balance clinical and academic responsibilities. Despite these barriers, general practice offers diverse opportunities for conducting clinical research. Clinical-academic GPs have the advantage of being embedded in a general practice, meaning that they understand the inner workings of their practice and patient population, giving them insight into the feasibility of specific research projects.

At our NHS primary care practice in the South West of England, we have successfully established a pro-research culture and developed a dedicated multidisciplinary clinical research team consisting of clinical-academic GPS, research nurses, a research healthcare assistant, a finance officer, and a dedicated non-GP clinical research physician. The recent appointment of a non-GP clinical research physician has expanded the team, enabling the practice to participate in more projects, pursue new areas of research, and develop independent research projects. To the best of our knowledge, we are the only NHS primary care practice in the South West of England to employ a dedicated non-GP clinical research physician. Based on our experience, we would recommend that other practices consider developing similar roles and infrastructure to facilitate meaningful participation in clinical research.

Creation of dedicated non-GP clinical research physician roles, embedded in NHS primary care practices, may be a way to integrate clinical research into this setting and build research capacity. Initiatives like this could address one of the key findings from a recent report, conducted by The Healthcare Improvement Studies (THIS) Institute, which explored how to involve NHS staff in research: 'Bringing a wider range of expertise into healthcare research may require new forms of career structures and building in time to conduct research beyond clinical academic and fellowship models. ${ }^{3}$

Rehan F Symonds,

GP, Oak Tree Surgery.

Email: r.symondsanhs.net

Samuel P Trethewey,

Clinical Research Physician, Oak Tree Surgery.

Kathryn J Beck,

Clinical Research Nurse, Oak Tree Surgery.

\section{Competing interests}

Dr Symonds is a senior GP partner at Oak Tree Surgery. Oak Tree Surgery is an RCGP 'Research Ready'-accredited NHS general practice and is a member of the Cornwall Clinical Research Group.

\section{REFERENCES}

1. Hobbs R. Is primary care research important and relevant to GPs? Br J Gen Pract 2019; DOI: https:// doi.org/10.3399/bjgp19X705149.

2. Royal College of General Practitioners. Fit for the Future: a vision for general practice. 2019. https:// unw.rcgp.org.uk/-/media/Files/News/2019/RCGP_ fit-for-the-future-report-may-2019.ashx?la=en (accessed 11 Mar 2020).

3. The Healthcare Improvement Studies (THIS) Institute. Involving NHS staff in research. 2019. https://wnw. thisinstitute.cam.ac.uk/research-articles/involvingnhs-staff-in-research (accessed 11 Mar 2020).

DOI: https://doi.org/10.3399/bjgp20X709313 